\title{
Oct4 and Sox 2 overexpression improves the proliferation and differentiation of bone mesenchymal stem cells in Xiaomeishan porcine
}

\author{
Y.X. Fan, C.H. Gu, Y.L. Zhang, B.S. Zhong, L.Z. Wang, Z.R. Zhou, \\ Z.Y. Wang, R.X. Jia and F. Wang \\ Jiangsu Livestock Embryo Engineering Laboratory, \\ Nanjing Agricultural University, Nanjing, China \\ Corresponding author: F. Wang \\ E-mail: caeet@njau.edu.cn
}

Genet. Mol. Res. 12 (4): 6067-6079 (2013)

Received April 12, 2013

Accepted September 19, 2013

Published December 2, 2013

DOI http://dx.doi.org/10.4238/2013.December.2.5

\begin{abstract}
Mesenchymal stem cells derived from bone marrow (BMSCs) are a population of self-renewing multipotent cells that are capable of differentiating into various cellular lineages, and are widely employed in tissue engineering and cell therapy. Recently, clinical research involving BMSCs has become increasingly popular. In order to conduct appropriate research, it is first necessary to amplify large amounts of functional BMSCs in vitro. However, after several passages of expanding in vitro, the proliferation and differentiation potential of BMSCs gradually decline. To determine whether overexpression of Oct4 or Sox 2 might prevent this decline, we transfected Oct4 or Sox2, which are essential for the pluripotency and self-renewal of embryonic stem cells, into BMSCs of Xiaomeishan porcine by a lentivirus. The results showed that overexpression of Sox 2 or Oct4 BMSCs in culture media containing a basic fibroblast growth factor resulted in higher proliferation
\end{abstract}


and differentiation compared to controls, suggesting that genetic modification of stemness-related genes is an efficient way to maintain the proliferation and differentiation potential of BMSCs.

Key words: Oct4; Sox2; Bone mesenchymal stem cells; Overexpression; Xiaomeishan porcine

\section{INTRODUCTION}

Bone mesenchymal stem cells (BMSCs) are a population of self-renewing multipotent cells. These stem cells are capable of differentiating into various cellular lineages, including the osteogenic, chondrogenic, adipogenic, and myogenic lineages (Pittenger et al., 1999; Jiang et al., 2002; Imabayashi et al., 2003; Bosch et al., 2006), and are widely used in clinical investigations (Friedenstein et al., 1966; Horwitz et al., 2002; Kadivar et al., 2006; Yang et al., 2008). Before human clinical trials can be approved, scaled-up cell production and delivery into an animal model with cell doses (number of cells) comparable to those anticipated for human trials are often required in order to satisfy regulatory safety concerns. Porcines display significant morphological and functional similarities to humans. Therefore, isolation and thorough characterization of porcine BMSCs offer great potential and would represent an advantageous experimental tool for tissue engineering and cell therapy. The main line of local Taihu pig in China, Xiaomeishan, is famous for its outstanding fecundity and meat quality, and is recognized worldwide for its outstanding genetic resources. Consequently, this breed is not only valuable for its resource conservation, but also for use in transgenic breeding to establish a culture of Xiaomeishan BMSCs. However, these cells tend to lose their proliferation and differentiation potential after several passages (Yoon et al., 2011). Furthermore, they enter the stages of senescence, characterized by enlarged and irregular cell shape, and cessation of cell division occurs after several passages (Hayflick, 1965).

The key transcription factors Oct4 and Sox 2 have been extensively studied in embryonic stem cells (ESCs). Oct4 is a critical transcription factor for regulating the selfrenewal and differentiation properties of ESCs, and is a POU domain transcription factor expressed in ESCs and germ cells (Nichols, et al., 1998). Overexpression of Oct4 causes ESC differentiation into primitive endoderm and mesoderm. Repression of Oct4 expression induces loss of ESC pluripotency and dedifferentiation to trophectoderm (Niwa et al., 2000). The transcription factor Sox2 is a member of the SRY-related HMG box family (Masui et al., 2007). It is expressed in pluripotent, embryonic, and extraembryonic cells and plays an important role in embryonic development and in maintaining the pluripotency and self-renewal ability of ESCs (Lefebvre et al., 2007; Niwa, 2007). Oct4 and Sox2 were shown to be critical factors for reprogramming somatic cells into induced pluripotent stem cells (Takahashi and Yamanaka, 2006). However, whether Oct4 or Sox2 genes might improve the growth and plasticity properties of BMSCs is still unknown.

In the current study, Oct4 and Sox 2 genes, essential for the pluripotency and selfrenewal of ESCs, were introduced into BMSCs of Xiaomeishan porcine, and their overexpressions were evaluated to determine whether they could improve the proliferation and differentiation abilities of BMSCs. 


\section{MATERIAL AND METHODS}

The experimental protocol was approved in accordance with the Guide for the Care and Use of Laboratory Animals prepared by the Ethics Committee of Nanjing Agricultural University.

\section{Isolation and culture of BMSCs}

Fresh bone marrow was extracted from Meishan porcine (2 weeks of age) femurs under sterile conditions. BMSCs were isolated by bone marrow aspiration by centrifugation $(20 \mathrm{~min}, 1500 \mathrm{~g})$ at room temperature. Then, cells were cultivated under standard culture conditions with Dulbecco's modified Eagle's medium with low glucose (L-DMEM; Gibco, USA) supplemented with $15 \%$ fetal bovine serum (FBS; Gibco), $100 \mathrm{U} / \mathrm{mL}$ penicillin, 100 $\mu \mathrm{g} / \mathrm{mL}$ streptomycin, and $2 \mathrm{mM}$ glutamine. Cultures were maintained at $37^{\circ} \mathrm{C}$ in a humidified atmosphere containing 5\% carbon dioxide. The medium was replaced after the first $72 \mathrm{~h}$ and then twice a week thereafter. Approximately 90-95\% confluence cells were subcultured at 3-5day intervals by trypsinization using $0.25 \%$ trypsin and $0.02 \%$ EDTA, and stored in freezing medium in liquid nitrogen.

\section{Flow cytometric analysis}

Phenotypic characterization of BMSCs was performed by flow cytometric analysis. Cells were trypsinized and washed with phosphate-buffered saline (PBS; Gibco) and incubated at $4^{\circ} \mathrm{C}$ for 30 min with the following cell-specific antibodies: CD29 (Abcam, USA), CD34 (Abcam), CD44 (AbD, England), CD45 (Abcam), and CD90 (Abcam). Cells were first permeabilized with cold methanol/PBS for $2 \mathrm{~min}$ at $-20^{\circ} \mathrm{C}$. After washing with cold PBS, cells were incubated with primary antibody $(1: 500)$ at $4^{\circ} \mathrm{C}$ for $30 \mathrm{~min}$, followed by staining with a fluorescein isothiocyanate (FITC)-linked secondary antibody (1:100) for an additional $30 \mathrm{~min}$. Labeled cell analysis was performed with an FACS Calibur cytometer (Becton Dickinson Immunocytometry System), and data were analyzed using the FlowJo software (Tree Star, Inc.)

\section{Lentivirus production and transduction}

293FT cells (Invitrogen, USA) were plated at $80 \%$ confluence and were transfected with the lentiviral vector of either Oct4 or Sox 2 along with packaging plasmids, using Lipofectamine 2000 (Invitrogen), following manufacturer instructions. The resulting supernatant was collected $48 \mathrm{~h}$ after transfection and filtered through a $0.45-\mu \mathrm{m}$ filter (Millipore, USA). The viruses were precipitated at $1500 \mathrm{~g}$ and resuspended with Opti-MEM medium (Invitrogen). Then, BMSCs were infected with either of the viruses to achieve Oct4 or Sox2 overexpression. BMSCs transfected with the empty vector, which was not inserted with Oct4 or Sox2, were used as controls. The expression of Oct4 and Sox 2 was detected by Western blotting.

\section{Western blot analysis}

Total cellular protein content of BMSCs with overexpressed Oct4, Sox2, and con- 
trol, and BMSCs at passage 3, were extracted using RIPA lysis buffer and incubated on ice for $30 \mathrm{~min}$. The extract was centrifuged at $12,000 \mathrm{~g}$ for $10 \mathrm{~min}$ at $4{ }^{\circ} \mathrm{C}$, the supernatant was collected, and the total protein content was measured using the bicinchoninic acid (BCA) assay. These proteins were separated using $0.1 \mathrm{~g} / \mathrm{mL}$ polyacrylamide sodium dodecyl sulfate (SDS) gel electrophoresis and transferred to polyvinylidene fluoride (PVDF) membranes, which were then incubated with a blocking buffer (Tris-buffered saline with $0.05 \mathrm{~g} / \mathrm{mL}$ milk and $0.1 \%(\mathrm{v} / \mathrm{v})$ Tween-20, $\mathrm{pH} 7.5)$ for $1 \mathrm{~h}$ at room temperature, and incubated overnight at $4^{\circ} \mathrm{C}$ with a monoclonal antibody against Oct 4 (1:100; Abcam) or Sox $2(1: 100$; Abcam). The mouse monoclonal antibody against $\alpha$-tubulin (1:2000; Santa Cruz) was used as a housekeeping control gene. The treated membranes were washed 3 times and incubated with the horseradish peroxidase-linked secondary antibody, which was diluted (1:8000) in the blocking buffer for $1 \mathrm{~h}$. Then, the blots were rinsed 3 times and detected for immunoreactivity by chemiluminescence detection, and were used to expose radiographic films to visualize immunoreactive signals. Finally, the blots were quantified by computerized densitometry using the Adobe Photoshop software (Adobe, Mountain View, CA, USA). Values were obtained using the anti-Oct4 or anti-Sox 2 antibody, which were normalized for the $\alpha$-tubulin present in the sample.

\section{Cell proliferation analysis}

The BMSCs with overexpressed Oct4 or Sox 2 and the control were respectively seeded on 24 -well plates $\left(1 \times 10^{4}\right.$ cells/well $)$ at passage 12 . Then, the cells were harvested by trypsinization at 24-h intervals for 7 days. Cell numbers were counted daily with a hemocytometer, and cell population growth was determined by calculating the population doubling time.

\section{Multipotential differentiation of BMSCs}

The BMSCs with the overexpressed Oct4 or Sox 2 and the control were cultured in LDMEM, containing $10 \%$ FBS until they reached $60-70 \%$ confluence, at which point they were induced by appropriate media to determine their multipotential differentiation abilities. To induce osteogenic differentiation, the BMSCs with overexpressed Oct4 and the control were each treated with osteogenic differentiation medium on 6 -well plates $\left(5 \times 10^{5}\right.$ cells/well $)$ for 2 weeks. The osteogenic differentiation medium consisted of DMEM supplemented with $10 \%$ FBS, $0.1 \mathrm{mM}$ dexamethasone (Sigma, USA), $10 \mathrm{mM}$ glycerol-2-phosphate (Sigma), and 50 $\mu \mathrm{g} / \mathrm{mL}$ ascorbic acid (Sigma). The BMSCs with overexpressed Sox2 were induced by osteogenic differentiation medium supplemented with basic fibroblast growth factor (bFGF). The culture medium was replaced with fresh medium twice a week. Adipogenic differentiation was induced on 6-well plates $\left(5 \times 10^{5}\right.$ cells/well) for 3 weeks by adipogenic differentiation medium consisting of DMEM supplemented with 10\% FBS, $1 \mathrm{mM}$ dexamethasone (Sigma), $0.5 \mathrm{mM}$ 3-isobutyl-1-methylxanthine (IBMX; Sigma), $10 \mathrm{mg} / \mathrm{mL}$ insulin (Sigma), and 0.1 $\mathrm{mM}$ indomethacin (Sigma). The culture medium was replaced with fresh medium twice a week. Differentiation of the BMSCs with overexpressed Oct4 or Sox2 and the control were evaluated by staining and real-time reverse transcription polymerase chain reaction (RT-PCR). Osteogenesis was stained with Alizarin red, and adipogenesis was examined with Oil Red O solution (Sigma). 


\section{RT-PCR analysis}

The differentiated BMSCs were harvested and washed once in PBS. Their total RNA was extracted using the PureLink RNA Mini kit (Invitrogen) according to manufacturer instructions, and reverse-transcribed using the Prime Script RT reagent kit (Takara, China). The mRNA levels were assayed with the SYBR Premix Ex Taq kit (Takara) on an ABI 7300 PRISM system (Applied Biosystems, USA). The amplification program consisted of an initial denaturation at $95^{\circ} \mathrm{C}$ for $30 \mathrm{~s}$ followed by 40 cycles at $95^{\circ} \mathrm{C}$ for $5 \mathrm{~s}$, annealing at $60^{\circ} \mathrm{C}$ for $31 \mathrm{~s}$, and extension at $72^{\circ} \mathrm{C}$ for $7 \mathrm{~min}$. RT-PCR was performed using the primers listed in Table 1. Each PCR analysis was performed in duplicate, with each set of assays repeated three times. To minimize the effects of unequal quantities of starting RNA and to eliminate potential sources of inconsistency, the relative expression levels of each gene were normalized to GAPDH using the $2^{-\Delta \Delta \mathrm{Ct}}$ method.

\begin{tabular}{|c|c|c|c|c|}
\hline Gene & Accession No. & Oligonucleotides $\left(5^{\prime} \rightarrow 3^{\prime}\right)($ up/down $)$ & Product size (bp) & Annealing temperature $\left({ }^{\circ} \mathrm{C}\right)$ \\
\hline$\overline{G A P D H}$ & AF017079 & $\begin{array}{l}\text { CTCAACGACCACTTCGTCAA } \\
\text { TCTGGGATGGAAACTGGAAG }\end{array}$ & 233 & 58 \\
\hline PPAR & AF059245 & $\begin{array}{l}\text { TGACCCAGAAAGCGATGC } \\
\text { ATGGCACTTTGGTAGTCCTG }\end{array}$ & 285 & 58 \\
\hline$C E B P-\alpha$ & AF103944 & $\begin{array}{l}\text { TGGACAAGAACA } \\
\text { TTGTCACTGGTCAGCTCCAG }\end{array}$ & 130 & 56 \\
\hline osteocalcin & AW346755 & $\begin{array}{l}\text { TCAACCCCGACTGCGACGAG } \\
\text { TTGGAGCAGCTGGGATGATGG }\end{array}$ & 204 & 62 \\
\hline osteonectin & NM001031794 & $\begin{array}{l}\text { CCATCGGCGAGTTTGAG } \\
\text { GCTTCTGCTTCTCGGTCA }\end{array}$ & 270 & 62 \\
\hline type $I \alpha I$ & AF201723 & $\begin{array}{l}\text { CCAAGAGGAGGGCCAAGAAGAAGG } \\
\text { GGGGCAGACGGGGCAGCACTC }\end{array}$ & 232 & 58 \\
\hline
\end{tabular}

\section{Statistical analysis}

All experiments were repeated at least three times. Values are reported as means \pm standard error of the mean (SE). The data were subjected to the independent sample Student $t$-test in the software package SPSS 17.0. Differences between means were determined by the Duncan multiple range test. Statistical differences were to be considered significant when $\mathrm{P}<0.05$.

\section{RESULTS}

\section{Characterization of BMSCs in vitro}

In this study, BMSCs of Xiaomeishan porcine were successfully established. Most of the nonadherent cells were removed at the first media replacement after $72 \mathrm{~h}$. Fibroblast-like cells attached to the plastic were evident at 3-5 days after initial seeding (Figure 1A). Most cell lines were composed of cells with a characteristic spindle shape, whereas the other lines had cells with a polygonal morphology (Figure 1B). The cells increased in number progressively and reached $80 \%$ confluence by $12-14$ days after seeding. However, after several passages, BMSCs entered senescence, characterized by enlarged and irregular cells (Figure 1C). 

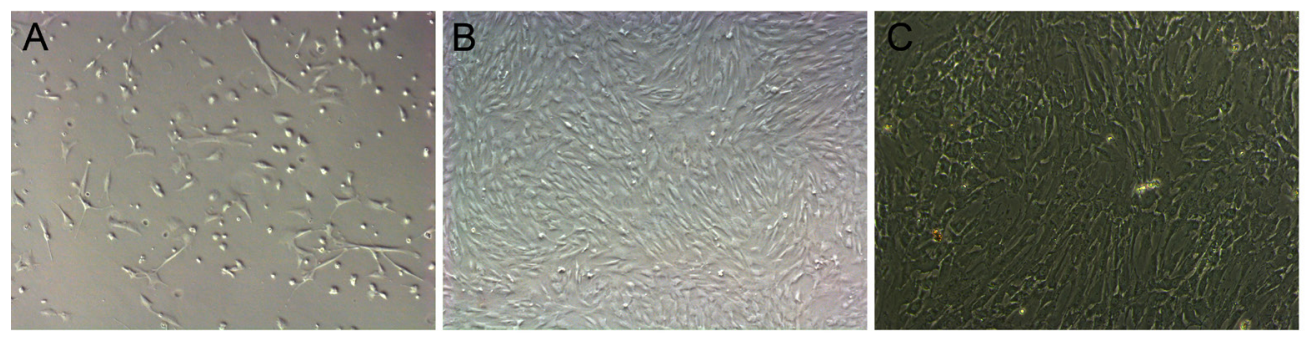

Figure 1. Morphology of Xiaomeishan porcine BMSCs. A. Cells exhibited a stretched fibroblast-like phenotype, mixed with some round-shaped erythrocytes and nonadherent cells at 6 days. B. Subcultivated cells, expanded up to at passage 3, presented a stable fibroblast-like morphology. C. BMSCs showed characteristic morphology changes after several passages, and became elongated in shape (100X).

To verify cell identity, Xiaomeishan porcine BMSCs were routinely examined by flow cytometry analysis at passage 3 . Cells were positive for the stromal markers CD29, CD44, and CD90, but not for the hematopoietic markers CD34 and CD45 (Figure 2).
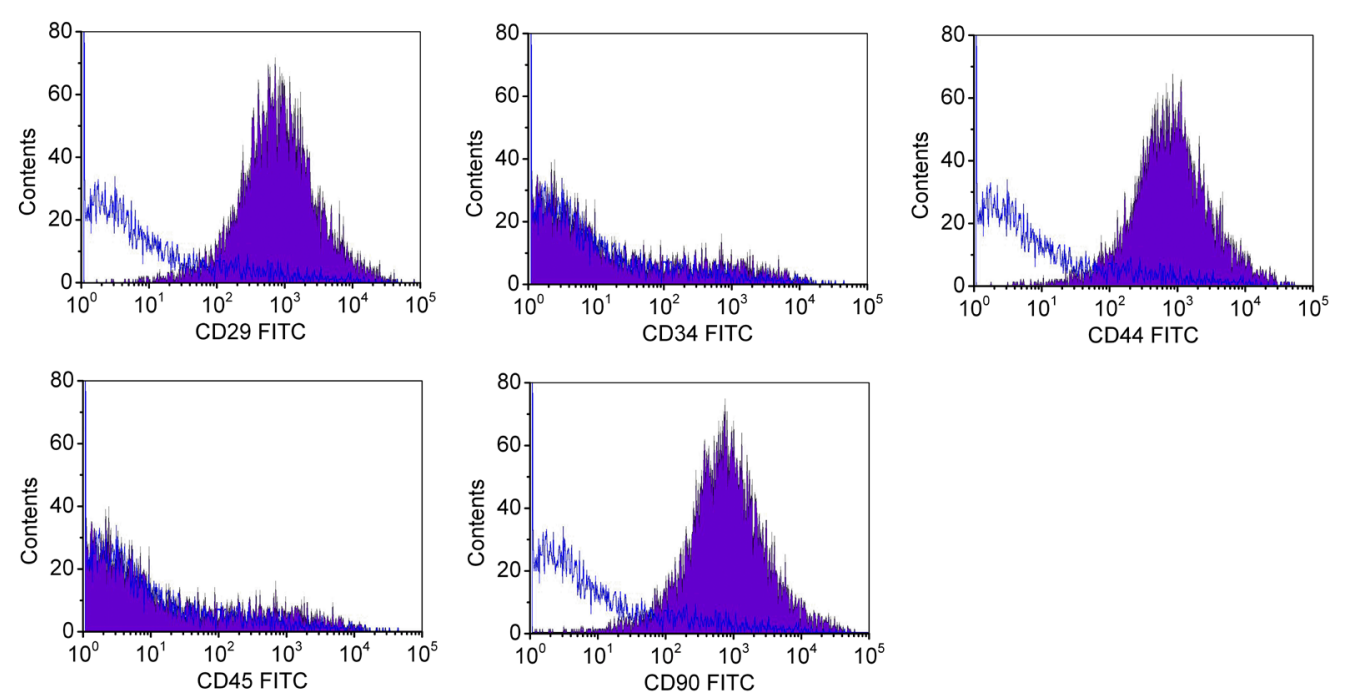

Figure 2. Flow cytometry histograms of three passaged Xiaomeishan porcine BMSCs for hematopoietic (CD34 and CD45) and stromal (CD29, CD44 and CD90) markers. BMSCs labeled with fluorescein isothiocyanate (FITC)-conjugated monoclonal antibodies. BMSCs were positive for CD29, CD44 and CD90, but negative for CD34 and CD45. The open areas stand for antibody isotype as control, and the shaded areas for the expression of the indicated markers.

\section{Oct4 or Sox2 expression in BMSCs}

In order to achieve high efficiency of introduction and subsequent stable expression of Oct 4 or Sox2 in BMSCs, a lentiviral vector was employed. The red fluorescent protein was used as a reporter gene, so that expression of the construct was easily detectable during the cell 
culture. The translation efficiency of Oct4 and Sox2 was high in BMSCs, and the fluorescent signal appeared strong under a fluorescence microscope (Figure 3). Western blotting results showed that the Oct4 protein content in Oct4-overexpressed BMSCs and the Sox 2 protein content in Sox2-overexpressed BMSCs at passage 3 were significantly higher $(\mathrm{P}<0.01)$ than protein contents in control or normal BMSCs. However, there was no difference in Oct4 or Sox 2 protein content between control and normal BMSCs $(\mathrm{P}>0.05)$ (Figure 4). Oct 4 or Sox2 was expressed effectively in the cells transfected with Oct4 or Sox2.

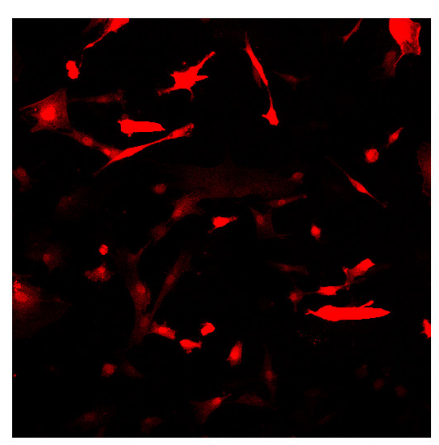

Control

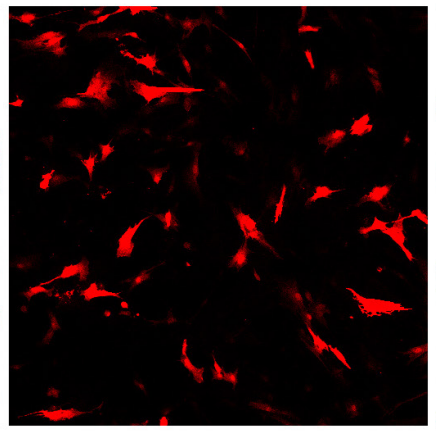

Oct4

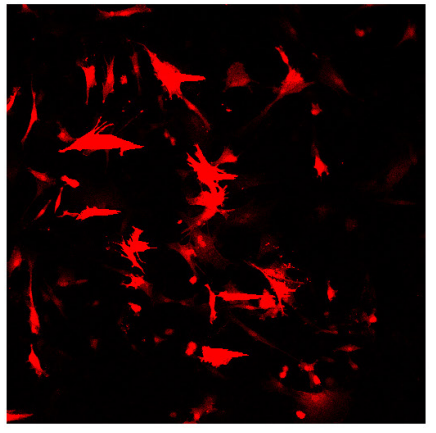

Sox2

Figure 3. Intensive red fluorescence was observed by fluorescence microscopy after lentivirus infecting for $48 \mathrm{~h}(100 \mathrm{X})$.

A

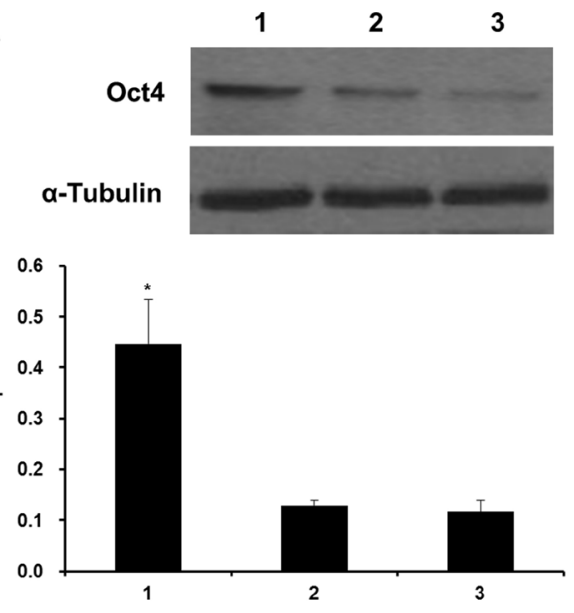

B
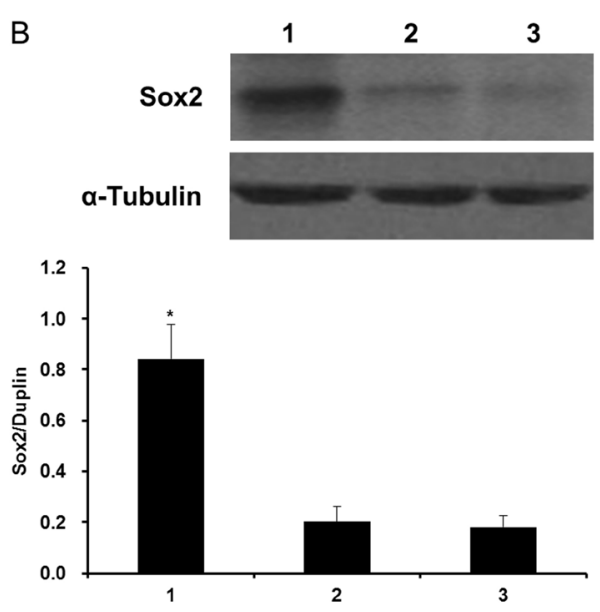

Figure 4. Detection of Oct4 (A) and Sox2 (B) protein expression by Western blot analysis and digital image analysis. Total cellular protein was extracted from Oct4- or Sox2-overexpressed BMSCs. The blot was first stained with the primary antibodies and then with horseradish peroxidase (HRP)-conjugated secondary antibody (goat antimouse IgG). The level of $\alpha$-tubulin protein was used as a loading control. Then, following immunoblot analysis, the intensities of Oct4 and Sox 2 immunoreactive bands were determined by digital image analysis. Data (ratio to $\alpha$-tubulin) represent means $\pm \mathrm{SE}$. Asterisks indicate a statistically significant difference. $\mathrm{P}<0.01$ vs 1 and 2 groups. Lane $1=$ Oct 4 or Sox 2 overexpressed BMSCs; lane 2 = control at passage 3; lane 3 = BMSCs at passage 3. 


\section{Growth properties of Oct4 or Sox 2 overexpression on BMSCs}

The proliferative ability of the Oct4- or Sox2-overexpressed BMSCs at passage 12 was compared with that of the control, which showed that Sox2-overexpressed cells had a higher proliferation potential in the presence of bFGF. However, the Oct4-overexpressed cells showed higher proliferation potential in the absence of bFGF (Figure 5A). During passage, the Oct4- and Sox2-overexpressed cells were relatively small and grew very well, whereas the control cells became elongated in shape (Figure 5B). Moreover, Sox2-overexpressed cells show higher proliferation potential in the presence of bFGF (Go et al., 2008). These observations suggested that Oct4 or Sox 2 overexpression not only promoted the proliferation of BMSCs, but also maintained the morphology of the cells.

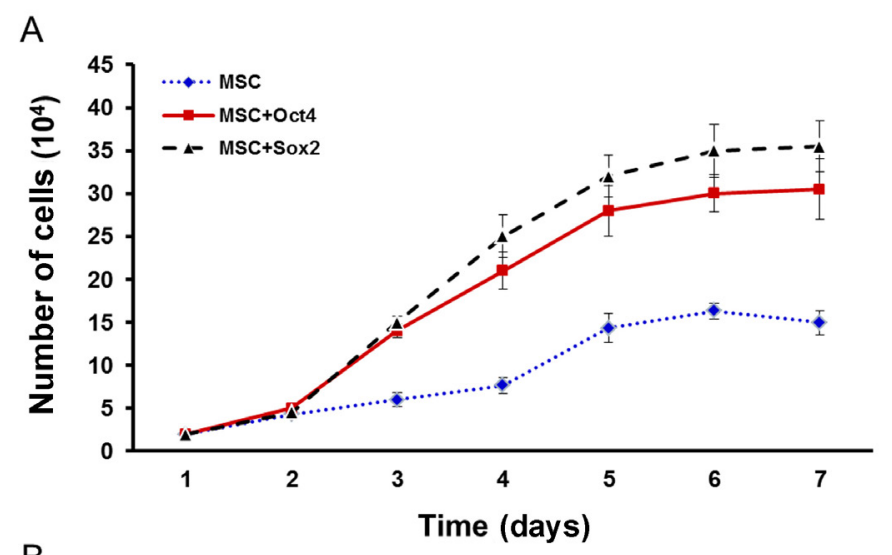

B

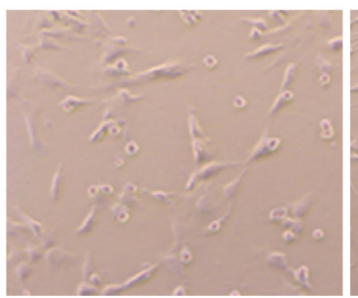

Control

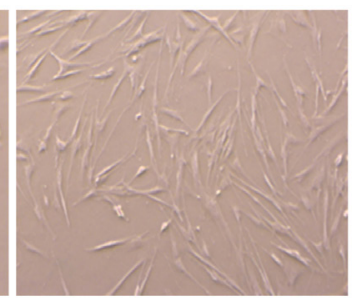

Oct4

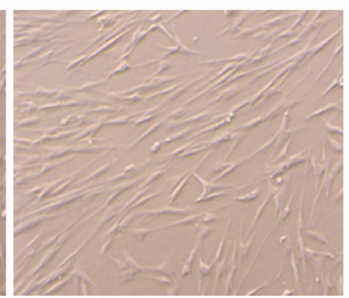

Sox2

Figure 5. Characterization of Oct4 and Sox2 overexpressing bone mesenchymal stem cells (BMSCs) and effects of their overexpression on proliferation of BMSCs. A. Proliferation curves of Oct4- or Sox2-overexpressed BMSCs compared with control. The time points represent the mean values from duplicate measurements and their standard deviation. Oct 4 or Sox 2 overexpression promoted cell growth of BMSCs. B. Oct 4 or Sox 2 overexpression maintained BMSCs morphology. At passage12, control became flat and gradually lost mesenchymal stem cell (MSC) morphology whereas Oct4 and Sox2 overexpression retained MSC morphology.

\section{Adipogenesis of Oct4 or Sox2-overexpressed BMSCs}

BMSCs can differentiate into adipocytes and osteoblasts. Therefore, the differentiation potential for adipoblasts of Oct4 or Sox2-expressing cells was examined. Oil Red O staining showed that Oct4 overexpression increased the number of lipid droplets at 21 days 
compared to the control (Figure 6A). Consistent with the staining result, Oct4 overexpression increased the adipogenic marker PPAR $\gamma$ by 1.94 -fold and $C E B P \alpha$ by 1.82 -fold compared with the control (Figure 6B). Similar to Oct4 overexpression, Oil Red O staining in adipogenesis showed that the adipogenesis of the Sox2-overexpressed BMSCs resulted in stronger lipid deposits than the control (Figure 6A). In the presence of bFGF, Sox2-overexpressed BMSCs raised the expression level of PPAR $\gamma$ by 2.25 -fold and $C E B P \alpha$ by 2.63 -fold (Figure 6B). These observations indicated that Oct4 or Sox 2 overexpression improved adipogenesis of BMSCs.

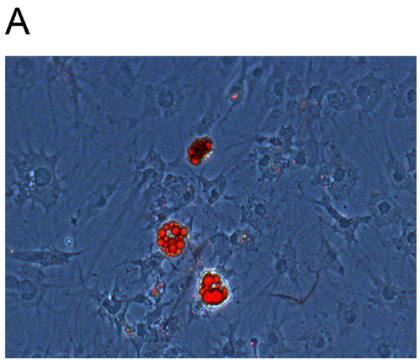

Control

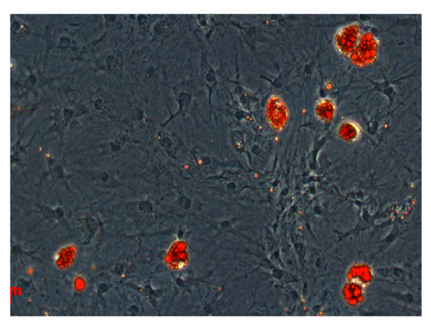

Oct4

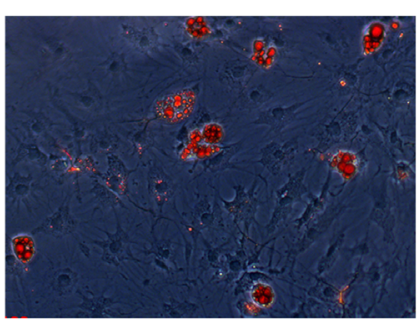

Sox2

B

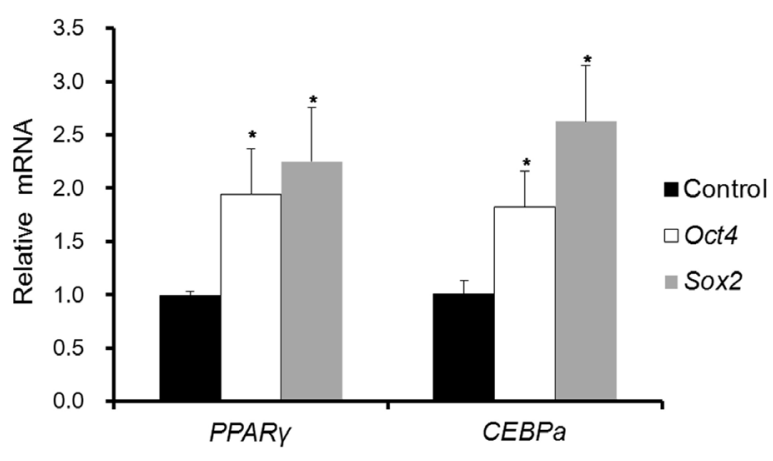

Figure 6. Adipogenic activities of Oct4- and Sox2-overexpressed BMSCs compared to control. A. Cells cultured in the adipoinductive medium for 3 weeks were stained by alkaline phosphatase activity. Oct 4 and Sox 2 overexpression improved adipogenesis of BMSCs. B. Cells cultured in adipoinductive medium for 3 weeks were collected and analyzed for the expression of $P P A R \gamma$ and $C E B P a$ transcripts using RT-PCR. Asterisks indicate significant differences compared to control $(\mathrm{P}<0.05)$.

\section{Osteogenesis of Oct4 or Sox2-overexpressed BMSCs}

The differentiation potentials for osteoblasts of Oct4 or Sox2-overexpressed cells were examined. The Oct4-overexpressed cells and the Sox2-overexpressed cells both showed higher osteogenic potentials compared to the control, as revealed by Alizarin red staining and by RT-PCR. Alizarin red staining showed that Oct4 overexpression raised the quantity of calcium deposition at 14 days compared to the control (Figure 7A). Consistent with the staining results, Oct 4 overexpression increased the expression level of type I 1 by 2.84 -fold, osteonectin by 1.56 -fold, and osteocalcin by 3.82-fold (Figure 7B). Therefore, both analyses showed 
that deposition of the mineralized matrix was enhanced by Oct4 overexpression (Figure 7A). These phenomena were also observed as a result of Sox 2 overexpression in which type I 1 , osteonectin, and osteocalcin expressions were upregulated by 3.83-, 1.97-, and 5.93-fold, respectively (Figure 7B). The Sox2-overexpressed cells showed higher osteogenesis potential in the presence of bFGF (Go et al., 2008). These observations indicated that Oct4 or Sox2 overexpression also improved the osteogenesis of BMSCs.

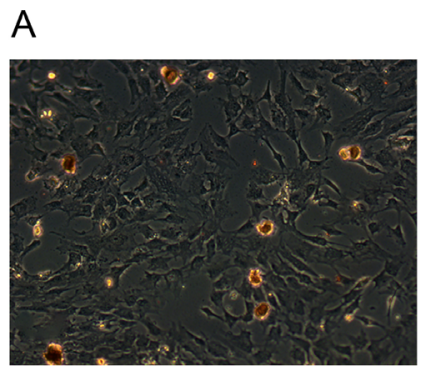

Control

B

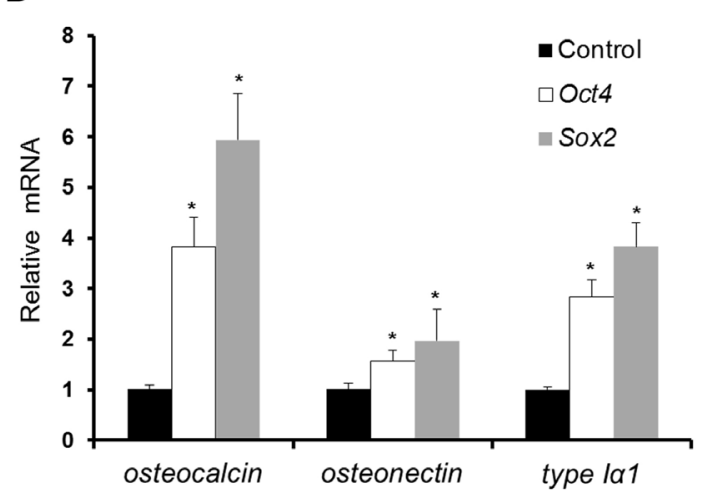

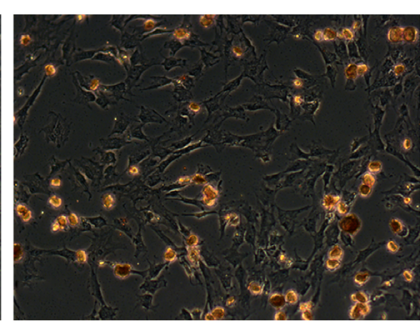

Oct4

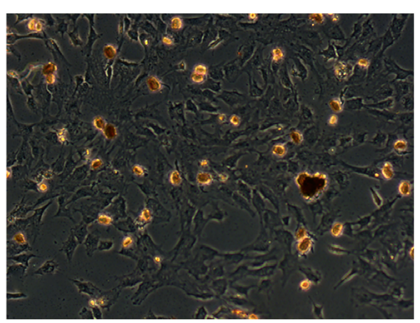

Sox2

Figure 7. Osteogenic activities of Oct4- and Sox2-expressed BMSCs compared to control. A. Cells cultured in the osteoinductive medium for 2 weeks were stained by Alizarin red. Oct4 and Sox 2 overexpression improved osteogenesis of BMSCs. B. Cells cultured in the osteoinductive medium for 2 weeks were collected and analyzed for the expression of osteocalcin, osteonectin, and type I 1 transcripts using RT-PCR. Asterisks indicate significant differences compared to control $(\mathrm{P}<0.05)$.

\section{DISCUSSION}

Mononuclear cells separated from porcine bone marrow were able to produce an adherent layer of cells when cultured in L-DMEM containing 15\% FBS in our culture system. These adherent cells were observed to be spindle and fibroblast-like under a phase-contrast light microscope. They were positive for the BMSC markers CD90, CD29, and CD44, and negative for CD45 and CD34, all of which were previously reported as MSC-related antigens (Pittenger et al., 1999).

MSCs derived from bone marrow have significant therapeutic potential with extensive applications for regenerative therapy. Based on previous observations, we speculated that 
BMSCs might represent a useful cell in clinical practices (Hu et al., 2010; Griffin et al., 2011; Gurudutta et al., 2012). Indeed, the advantage of employing large animals, such as pigs, rather than small animal models, is based on several factors, including disease complexity, effective cell dosage, cell survival after transplantation, as well as tissue related inflammatory and immunological responses to engraftment after transplantation (Poncelet et al., 2007; Kuo et al., 2009). Xiaomeishan porcine BMSCs were chosen for this study based on their similarity to human cells. Therefore, studies using the Xiaomeishan porcine model may offer significant contributions toward the development of cellular therapy strategies for a clinical setting. In vivo tracking studies to examine the survival, distribution, and homing of BMSCs after infusion in large animal models should further allow optimization of the clinical use of BMSCs to promote tolerance induction in solid organ transplantation patients. In addition, this is the first study to examine the effect of pluripotent genes on proliferation and differentiation of BMSCs from Xiaomeishan porcine.

The expansion and differentiation potential of these BMSCs in vitro is essential for use in various stem cell therapies. However, the cells lose their proliferation and differentiation abilities after several passages. Moreover, little is known about how self-renewal and differentiation are regulated in BMSCs at present.

To this end, the present study focused on the reported expression of the embryonic transcription factors Oct4 and Sox 2 in BMSCs. Oct4 and Sox2 are transcription factors known to be essential for normal pluripotent cell development and maintenance (Dailey and Basilico, 2001; Rodda et al., 2005). Oct4 is a critical transcription factor for regulating self-renewal and differentiation of ESCs. In the absence of Oct4, ESCs revert to the trophoblast lineage in vivo and in vitro. Increasing the expression of Oct4 above endogenous levels in ESCs leads to differentiation toward the extraembryonic mesoderm lineage (Niwa et al., 2000). Based on previous observations, we observed cell growth and found that overexpression of Oct4 promoted the proliferative rate and differentiation potential. In addition, Oct4-overexpressed BMSCs were relatively small with a spindle-shaped morphology and grew very well. Therefore, Oct4 overexpression played a role in maintaining the stemness of Xiaomeishan porcine BMSCs.

Sox2 is transcription factor involved in the regulation of stem cell development and in the determination of cell fate. Recently, a novel function for Sox 2 was identified in a mouse osteoblastic lineage: the maintenance of the self-renewal of cells. In addition, Sox2-knockout cells could not form colonies, and their population growth was arrested with a senescent phenotype (Basu-Roy et al., 2010). In another study, most Sox2-overexpressed BMSCs were small and showed high proliferative and osteogenic capabilities from human BMSCs (Go et al., 2008). Knockdowns of Sox2 and Msx2 using RNA interference, showed a significant decrease in colony-forming ability. Specifically, a knockdown of Sox 2 significantly inhibited multipotentiality and cell proliferation (Yoon et al., 2011). Overall, our findings were in good accordance with those of previous studies. Sox2-overexpressed cells all showed significantly higher viability and differentiation potential compared to control cells, and these cells were smaller with a spindle-shaped morphology. These findings suggested that Sox2 overexpression maintained the stemness of Xiaomeishan porcine BMSCs and might function in a similar fashion in progenitor BMSCs as in ESCs.

The overexpression of Oct4 and Sox2 was detected in BMSCs, but at lower levels than has been observed in ESCs (Wei and Shen, 2011). Moreover, although the expression of Oct4 was observed in early-passage MSCs, its expression was too low to detect at late-passage 
(Greco et al., 2007). Therefore, it is possible that low-level Oct4 and Sox2 underwent growth arrest or a program of differentiation. Oct4 expression could be an indicator of MSC differentiation potential, which could be used as an experimental tool or in clinical diagnostics.

Pluripotent stem cells can be directly generated by reprogramming somatic cells using defined transcription factors, including Oct4 and Sox2 (Takahashi and Yamanaka, 2006; Qin et al., 2007). Therefore, Oct4 and Sox2 are critical for cell reprogramming (Takahashi and Yamanaka, 2006; Qin et al., 2007) and for altering the cell status. Our observations on the overexpression of Oct4 or Sox2 in Xiaomeishan porcine BMSCs were consistent with those of previous studies. We succeeded in maintaining the proliferation and differentiation potentials of cells in senescent passages by introducing Oct4 or Sox2, which could be an effective and feasible way of maintaining high-quality Xiaomeishan porcine BMSCs as stem cells.

\section{ACKNOWLEDGMENTS}

Research supported by the National Natural Science Foundation of China (\#31201802). We are grateful to Prof. Feirong Gu of the College of Foreign Studies, NJAU, for language editing of this manuscript. We also thank Shijia Ying and Hui Song for their excellent work and Changlong Wang and Haitao Nie for their help in the collection of the experimental materials.

\section{REFERENCES}

Basu-Roy U, Ambrosetti D, Favaro R, Nicolis SK, et al. (2010). The transcription factor Sox2 is required for osteoblast self-renewal. Cell Death Differ. 17: 1345-1353.

Bosch P, Pratt SL and Stice SL (2006). Isolation, characterization, gene modification, and nuclear reprogramming of porcine mesenchymal stem cells. Biol. Reprod. 74: 46-57.

Dailey L and Basilico C (2001). Coevolution of HMG domains and homeodomains and the generation of transcriptional regulation by Sox/POU complexes. J. Cell Physiol. 186: 315-328.

Friedenstein AJ, Piatetzky-Shapiro II and Petrakova KV (1966). Osteogenesis in transplants of bone marrow cells. $J$. Embryol. Exp. Morphol. 16: 381-390.

Go MJ, Takenaka C and Ohgushi H (2008). Forced expression of Sox2 or Nanog in human bone marrow derived mesenchymal stem cells maintains their expansion and differentiation capabilities. Exp. Cell Res. 314: 1147-1154.

Greco SJ, Liu K and Rameshwar P (2007). Functional similarities among genes regulated by OCT4 in human mesenchymal and embryonic stem cells. Stem. Cells 25: 3143-3154.

Griffin M, Iqbal SA and Bayat A (2011). Exploring the application of mesenchymal stem cells in bone repair and regeneration. J. Bone Joint Surg. Br. 93: 427-434.

Gurudutta GU, Satija NK, Singh VK, Verma YK, et al. (2012). Stem cell therapy: a novel \& futuristic treatment modality for disaster injuries. Indian J. Med. Res. 135: 15-25.

Hayflick L (1965). The limited in vitro lifetime of human diploid cell strains. Exp. Cell Res. 37: 614-636.

Horwitz EM, Gordon PL, Koo WK, Marx JC, et al. (2002). Isolated allogeneic bone marrow-derived mesenchymal cells engraft and stimulate growth in children with osteogenesis imperfecta: Implications for cell therapy of bone. Proc. Natl. Acad. Sci. U. S. A. 99: 8932-8937.

Hu YL, Fu YH, Tabata Y and Gao JQ (2010). Mesenchymal stem cells: a promising targeted-delivery vehicle in cancer gene therapy. J. Control Release 147: 154-162.

Imabayashi H, Mori T, Gojo S, Kiyono T, et al. (2003). Redifferentiation of dedifferentiated chondrocytes and chondrogenesis of human bone marrow stromal cells via chondrosphere formation with expression profiling by large-scale cDNA analysis. Exp. Cell Res. 288: 35-50.

Jiang Y, Jahagirdar BN, Reinhardt RL, Schwartz RE, et al. (2002). Pluripotency of mesenchymal stem cells derived from adult marrow. Nature 418: 41-49.

Kadivar M, Khatami S, Mortazavi Y, Shokrgozar MA, et al. (2006). In vitro cardiomyogenic potential of human umbilical vein-derived mesenchymal stem cells. Biochem. Biophys Res. Commun. 340: 639-647.

Kuo YR, Goto S, Shih HS, Wang FS, et al. (2009). Mesenchymal stem cells prolong composite tissue allotransplant 
survival in a swine model. Transplantation 87: 1769-1777.

Lefebvre V, Dumitriu B, Penzo-Mendez A, Han Y, et al. (2007). Control of cell fate and differentiation by Sry-related high-mobility-group box (Sox) transcription factors. Int. J. Biochem. Cell Biol. 39: 2195-2214.

Masui S, Nakatake Y, Toyooka Y, Shimosato D, et al. (2007). Pluripotency governed by Sox2 via regulation of Oct3/4 expression in mouse embryonic stem cells. Nat. Cell Biol. 9: 625-635.

Nichols J, Zevnik B, Anastassiadis K, Niwa H, et al. (1998). Formation of pluripotent stem cells in the mammalian embryo depends on the POU transcription factor Oct4. Cell 95: 379-391.

Niwa H (2007). How is pluripotency determined and maintained? Development 134: 635-646.

Niwa H, Miyazaki J and Smith AG (2000). Quantitative expression of Oct-3/4 defines differentiation, dedifferentiation or self-renewal of ES cells. Nat. Genet. 24: 372-376.

Pittenger MF, Mackay AM, Beck SC, Jaiswal RK, et al. (1999). Multilineage potential of adult human mesenchymal stem cells. Science 284: 143-147.

Poncelet AJ, Vercruysse J, Saliez A and Gianello P (2007). Although pig allogeneic mesenchymal stem cells are not immunogenic in vitro, intracardiac injection elicits an immune response in vivo. Transplantation 83: 783-790.

Qin D, Li W, Zhang J and Pei D (2007). Direct generation of ES-like cells from unmodified mouse embryonic fibroblasts by Oct4/Sox2/Myc/Klf4. Cell Res. 17: 959-962.

Rodda DJ, Chew JL, Lim LH, Loh YH, et al. (2005). Transcriptional regulation of nanog by OCT4 and SOX2. J. Biol. Chem. 280: 24731-24737.

Takahashi K and Yamanaka S (2006). Induction of pluripotent stem cells from mouse embryonic and adult fibroblast cultures by defined factors. Cell 126: 663-676.

Wei X and Shen CY (2011). Transcriptional regulation of oct4 in human bone marrow mesenchymal stem cells. Stem Cells Dev. 20: 441-449.

Yang YJ, Qian HY, Huang J, Geng YJ, et al. (2008). Atorvastatin treatment improves survival and effects of implanted mesenchymal stem cells in post-infarct swine hearts. Eur. Heart J. 29: 1578-1590.

Yoon DS, Kim YH, Jung HS, Paik S, et al. (2011). Importance of Sox2 in maintenance of cell proliferation and multipotency of mesenchymal stem cells in low-density culture. Cell Prolif. 44: 428-440. 